\title{
Charge Modulations in the Superconducting State of the Cuprates
}

\author{
Degang Zhang \\ Texas Center for Superconductivity and Department of Physics, University of Houston, Houston, TX 77204, USA
}

\begin{abstract}
Motivated by the recent scanning tunneling microscopy (STM) and neutron scattering experiments, we investigate various charge density wave orders coexisting with superconductivity in the cuprate superconductors. The explicit expressions of the local density of states and its Fourier component at the ordering wavevector for the weak charge modulations are derived. It is shown that the STM experiments in $\mathrm{Bi}_{2} \mathrm{Sr}_{2} \mathrm{CaCu}_{2} \mathrm{O}_{8+\delta}$ cannot be explained by a site- or bond-centered charge modulation alone, but agree well with the presence of the dimerization hopping and transverse pairing modulations. We also calculate the spectral function for the charged stripes, which is measured by the ARPES experiments.
\end{abstract}

The coexistence of charged stripes and superconductivity in high- $T_{c}$ superconductors has attracted a lot of both experimental and theoretical attention recently. In a novel STM experiment, Hoffman et al observed a four cell checkbroad local density of states (LDOS) modulation around the cores of superconducting vortices in $\mathrm{Bi}_{2} \mathrm{Sr}_{2} \mathrm{CaCu}_{2} \mathrm{O}_{8+\delta}$ by applying a magnetic field [1]. The charge modulation occurs in the $\mathrm{Cu}-\mathrm{O}$ bond direction and in the energy range $0 \leq E \leq 12 \mathrm{meV}$. Very recently Howald et al discovered similar charge modulation at energy $E=25 \mathrm{meV}$ but in absence of magnetic field [2]. The neutron scattering experiments on underdoped $\mathrm{YBa}_{2} \mathrm{Cu}_{3} \mathrm{O}_{6.35}$ have also shown the charge density wave order with a period of eight lattice constants coexisting with superconductivity [3].

To explain the STM spectra in $\mathrm{Bi}_{2} \mathrm{Sr}_{2} \mathrm{CaCu}_{2} \mathrm{O}_{8+\delta}$, a number of theoretical studies based on different models have been carried out by various authors [4-10]. However, it is important to determine the correct charge density wave orders in order to understand the origin of the charge stripes. In Ref. [8], Podolsky et al analysed in detail the influence of various patterns of translational symmetry breaking on the Fourier component of LDOS at the ordering wavevector $\mathbf{Q}$ by emyloping an approximate technique. They concluded that the STM experiments [2] are consistent with the periodic modulation in the electron hopping. In contrast, by the numerical simulation of a d-wave superconductor with two-dimensional site charge density wave, bond charge density wave or pairing modulation, the pairing amplitude modulation comes closest to the experimental curves of Ref. [2] [9]. Therefore, there is no consensus about the constitution of the charged stripes. In this paper, in order to explain well the STM experiments, we solve strictly the d-wave superconductor with one-dimensional weak charge modulations. The results show that the dimerization hopping and transverse pairing modulations are consistent with the STM experiments. We also discuss the ARPES experiments on the charged stripes, which verify the existence of the hopping and pairing modulations.

We start from the mean-field Hamiltonian of a d-wave superconductor

$$
H_{\mathrm{BCS}}=\sum_{\mathbf{K} \sigma} \epsilon_{\mathbf{K}} c_{\mathbf{K} \sigma}^{\dagger} c_{\mathbf{K} \sigma}+\sum_{\mathbf{K}} \Delta_{\mathbf{K}}\left(c_{\mathbf{K} \uparrow}^{\dagger} c_{-\mathbf{K} \downarrow}^{\dagger}+c_{-\mathbf{K} \downarrow} c_{\mathbf{K} \uparrow}\right)
$$

where $\epsilon_{\mathbf{K}}=t_{0}+t_{1}\left(\cos K_{x}+\cos K_{y}\right) / 2+t_{2} \cos K_{x} \cos K_{y}+$ $t_{3}\left(\cos 2 K_{x}+\cos 2 K_{y}\right) / 2+t_{4}\left(\cos 2 K_{x} \cos K_{y}+\right.$ $\left.\cos K_{x} \cos 2 K_{y}\right) / 2+t_{5} \cos 2 K_{x} \cos 2 K_{y}$ and $\Delta_{\mathbf{K}}=$ $\Delta_{0}\left(\cos K_{x}-\cos K_{y}\right) / 2$. For nearly optimally doped $\mathrm{Bi}_{2} \mathrm{Sr}_{2} \mathrm{CaCu}_{2} \mathrm{O}_{8+\delta}, \quad t_{0-5}=$ $0.1305,-0.5951,0.1636,-0.0519,-0.1117,0.0510(\mathrm{eV})$

[11]. For underdoped $\mathrm{YBa}_{2} \mathrm{Cu}_{3} \mathrm{O}_{6.35}, t_{0-2}=$ $0.2445,-1.2000,0.3600(\mathrm{eV})$ and $t_{3-5}=0.0(\mathrm{eV})(6$ percent doping). For both superconductors, we choose $\Delta_{0}=0.0400 \mathrm{eV}$.

The charge modulations can be introduced phenomenologically into the Hamiltonian (1) by adding the charge ordered parameters, which have a general form

$$
\begin{aligned}
H_{\mathbf{C}}= & \sum_{\mathbf{K} \sigma}\left(f_{\mathbf{K}} c_{\mathbf{K}+\mathbf{Q} \sigma}^{\dagger} c_{\mathbf{K} \sigma}+f_{\mathbf{K}}^{*} c_{\mathbf{K} \sigma}^{\dagger} c_{\mathbf{K}+\mathbf{Q} \sigma}\right) \\
& +\sum_{\mathbf{K}}\left(g_{\mathbf{K}} c_{\mathbf{K}+\mathbf{Q} \uparrow}^{\dagger} c_{-\mathbf{K} \downarrow}^{\dagger}+g_{\mathbf{K}}^{*} c_{\mathbf{K} \uparrow}^{\dagger} c_{-\mathbf{K}-\mathbf{Q} \downarrow}^{\dagger}+h . c\right),
\end{aligned}
$$

where $\mathbf{Q}=\mathrm{Qe}_{x}\left(\mathrm{Q}=\pi / 2\right.$ for $\mathrm{Bi}_{2} \mathrm{Sr}_{2} \mathrm{CaCu}_{2} \mathrm{O}_{8+\delta}$ and $\pi / 4$ for $\left.Y B a_{2} C u_{3} O_{6.35}\right), f_{\mathbf{K}}\left(\equiv f_{-\mathbf{K}-\mathbf{Q}}\right)$ and $g_{\mathbf{K}}(\equiv$ $g_{-\mathbf{K}-\mathbf{Q})}$ describe the hopping modulations and pairing modulations, respectively. We note that $f_{\mathbf{K}}=$ $\lambda_{1}, \lambda_{2} e^{-i \mathrm{Q} / 2}, \lambda_{3} \cos \left(K_{x}+\mathrm{Q} / 2\right) e^{-i \mathrm{Q} / 2}$ and $\lambda_{4} \cos K_{y}$ are the site-centered, bond-centered, longitudinal dimerization and transverse dimerization charge modulations while $g_{\mathbf{K}}=\lambda_{5} \cos \left(K_{x}+\mathrm{Q} / 2\right) e^{-i \mathrm{Q} / 2}$ and $\lambda_{6} \cos K_{y}$ are the longitudinal and transverse pairing modulations [8]. In this paper, we restrict our discussion to the case of weak charge modulations, i. e. small $\lambda$ 's and do not consider the influence of the incommensurate spin orders to LDOS. In fact, the spin orders were not observed experimentally [2].

Taking the Bogoliubov transformation

$$
\begin{aligned}
& c_{\mathbf{k}+m \mathbf{Q} \uparrow}=\xi_{\mathbf{k} m 0} \psi_{\mathbf{k} m 0}-\xi_{\mathbf{k} m 1} \psi_{\mathbf{k} m 1} \\
& c_{-\mathbf{k}-m \mathbf{Q} \downarrow}^{\dagger}=\xi_{\mathbf{k} m 1} \psi_{\mathbf{k} m 0}+\xi_{\mathbf{k} m 0} \psi_{\mathbf{k} m 1},
\end{aligned}
$$

where $\mathbf{k}$ is restricted to the reduced Brillouin zone, $m=0,1,2, \cdots, N=(2 \pi / \mathrm{Q})-1, \xi_{\mathbf{k} m \nu}^{2}=\frac{1}{2}[1+$ $\left.(-1)^{\nu} \epsilon_{\mathbf{k}+m \mathbf{Q}} / E_{\mathbf{k}+m \mathbf{Q}}\right], \quad \xi_{\mathbf{k} m 0} \xi_{\mathbf{k} m 1}=\Delta_{\mathbf{k}+m \mathbf{Q}} / 2 E_{\mathbf{k}+m \mathbf{Q}}$ 
and $E_{\mathbf{k}+m \mathbf{Q}}=\left(\epsilon_{\mathbf{k}+m \mathbf{Q}}^{2}+\Delta_{\mathbf{k}+m \mathbf{Q}}^{2}\right)^{\frac{1}{2}}$, the total Hamiltonian $H=H_{\mathrm{BCS}}+H_{\mathrm{C}}$ can be rewritten as

$$
\begin{gathered}
H=\sum_{\mathbf{k} m \nu}(-1)^{\nu} E_{\mathbf{k}+m \mathbf{Q}} \psi_{\mathbf{k} m \nu}^{\dagger} \psi_{\mathbf{k} m \nu} \\
+\sum_{\mathbf{k} m \nu \nu^{\prime}}\left\{\left[\alpha_{m+1 m}^{\nu \nu^{\prime}}(\mathbf{k})+\beta_{m+1 m}^{\nu \nu^{\prime}}(\mathbf{k})\right] \psi_{\mathbf{k} m+1 \nu}^{\dagger} \psi_{\mathbf{k} m \nu^{\prime}}+h . c\right\},
\end{gathered}
$$

where $\alpha_{m+1 m}^{\nu \nu^{\prime}}(\mathbf{k})=f_{\mathbf{k}+m \mathbf{Q}}\left[(-1)^{\nu+\nu^{\prime}} \xi_{\mathbf{k} m+1 \nu} \xi_{\mathbf{k} m \nu^{\prime}}-\right.$ $\left.\xi_{\mathbf{k} m+1 \nu+1} \xi_{\mathbf{k} m \nu^{\prime}+1}\right]$ and $\beta_{m+1 m}^{\nu \nu^{\prime}}(\mathbf{k})=g_{\mathbf{k}+m \mathbf{Q}}\left[(-1)^{\nu} \xi_{\mathbf{k} m+1 \nu}\right.$ $\left.\times \xi_{\mathbf{k} m \nu^{\prime}+1}+(-1)^{\nu^{\prime}} \xi_{\mathbf{k} m+1 \nu+1} \xi_{\mathbf{k} m \nu^{\prime}}\right]$.

We define two-point Green's functions

$$
G_{m m^{\prime}}^{\nu \nu^{\prime}}\left(\mathbf{k}, \mathbf{k}^{\prime} ; i \omega\right)=-\mathcal{F}<T_{\tau}\left[\psi_{\mathbf{k} m \nu}(\tau) \psi_{\mathbf{k}^{\prime} m^{\prime} \nu^{\prime}}^{\dagger}(0)\right]>,
$$

where $\mathcal{F} \phi(\tau)$ denote the Fourier transform of $\phi(\tau)$ in Matsubara frequencies. Then the equations of motion for the Green's functions are

$$
\begin{gathered}
{\left[i \omega_{n}-(-1)^{\nu} E_{\mathbf{k}+m \mathbf{Q}}\right] G_{m m^{\prime}}^{\nu \nu^{\prime}}\left(\mathbf{k}, \mathbf{k}^{\prime} ; i \omega_{n}\right)} \\
-\sum_{\nu^{\prime \prime}}(-1)^{\nu+\nu^{\prime \prime}} \xi_{\mathbf{k} m \nu+\nu^{\prime \prime}}\left[f_{\mathbf{k}+(m-1) \mathbf{Q}} \mathcal{G}_{m-1 m^{\prime \prime}}^{\nu^{\prime \prime} \nu^{\prime}}\left(\mathbf{k}, \mathbf{k}^{\prime} ; i \omega_{n}\right)\right. \\
\left.+f_{\mathbf{k}+m \mathbf{Q}}^{*} \mathcal{G}_{m+1 m^{\prime}}^{\nu^{\prime \prime} \nu^{\prime}}\left(\mathbf{k}, \mathbf{k}^{\prime} ; i \omega_{n}\right)\right] \\
-\sum_{\nu^{\prime \prime}}(-1)^{\nu \nu^{\prime \prime}} \xi_{\mathbf{k} m \nu+\nu^{\prime \prime}+1}\left[g_{\mathbf{k}+(m-1) \mathbf{Q}} \mathcal{G}_{m-1 m^{\prime}}^{\nu^{\prime \prime} \nu^{\prime}}\left(\mathbf{k}, \mathbf{k}^{\prime} ; i \omega_{n}\right)\right. \\
\left.+g_{\mathbf{k}+m \mathbf{Q}}^{*} \mathcal{G}_{m+1 m^{\prime}}^{\nu^{\prime \prime} \nu^{\prime}}\left(\mathbf{k}, \mathbf{k}^{\prime} ; i \omega_{n}\right)\right]=\delta_{\mathbf{k k}^{\prime}} \delta_{m m^{\prime}} \delta_{\nu \nu^{\prime}}
\end{gathered}
$$

where

$$
\begin{aligned}
\mathcal{G}_{m m^{\prime}}^{\nu \nu^{\prime}}\left(\mathbf{k}, \mathbf{k}^{\prime} ; i \omega_{n}\right) & =\sum_{\nu_{m m^{\prime}}}\left(\mathbf{k}, \mathbf{k}^{\prime \prime} ; i \omega_{n}\right) \\
& \times G^{\nu^{\prime \prime} \nu^{\prime}}
\end{aligned}
$$

Obviously, combining Eqs.(6) and (7), the anomalous Green's functions in Eq. (5) can be solved by inverting a $2(N+1) \times 2(N+1)$ matrix. To this end, we define

$$
\begin{gathered}
G_{m \nu}^{0}\left(\mathbf{k} ; i \omega_{n}\right)=\frac{1}{i \omega_{n}-(-1)^{\nu} E_{\mathbf{k}+m \mathbf{Q}}}, \\
a_{m}\left(\mathbf{k} ; i \omega_{n}\right)=\sum_{\nu} \xi_{\mathbf{k} m \nu}^{2} G_{m \nu}^{0}\left(\mathbf{k} ; i \omega_{n}\right), \\
b_{m}\left(\mathbf{k} ; i \omega_{n}\right)=\sum_{\nu}(-1)^{\nu} \xi_{\mathbf{k} m \nu} \xi_{\mathbf{k} m \nu+1} G_{m \nu}^{0}\left(\mathbf{k} ; i \omega_{n}\right), \\
c_{m}\left(\mathbf{k} ; i \omega_{n}\right)=\sum_{\nu} \xi_{\mathbf{k} m \nu+1}^{2} G_{m \nu}^{0}\left(\mathbf{k} ; i \omega_{n}\right), \\
g_{m m^{\prime}}^{\nu \nu^{\prime}}\left(\mathbf{k}, \mathbf{k}^{\prime} ; i \omega_{n}\right)=(-1)^{(1-\nu) \nu^{\prime}} \delta_{\mathbf{k k}^{\prime}} \delta_{m m^{\prime}} \xi_{\mathbf{k} m \nu+\nu^{\prime}} \\
\times G_{m \nu^{\prime}}^{0}\left(\mathbf{k} ; i \omega_{n}\right),
\end{gathered}
$$

and introduce the $2(N+1)$ vectors $\mathcal{G}$ and $\mathbf{g}$

$$
\begin{gathered}
\mathcal{G}=\left(\begin{array}{c}
\mathcal{G}^{0} \\
\mathcal{G}^{1}
\end{array}\right), \mathcal{G}^{0}=\left(\begin{array}{c}
\mathcal{G}_{0 m^{\prime}}^{0 \nu^{\prime}} \\
\vdots \\
\mathcal{G}_{N \nu^{\prime}}^{0 \nu^{\prime}}
\end{array}\right), \mathcal{G}^{1}=\left(\begin{array}{c}
\mathcal{G}_{0 m^{\prime}}^{1 \nu^{\prime}} \\
\vdots \\
\mathcal{G}_{N \nu^{\prime}}^{1 \nu^{\prime}}
\end{array}\right), \\
\mathbf{g}=\left(\begin{array}{c}
\mathbf{g}^{0} \\
\mathbf{g}^{1}
\end{array}\right), \mathbf{g}^{0}=\left(\begin{array}{c}
g_{0 m^{\prime}}^{1 \nu^{\prime}} \\
\vdots \\
g_{N m^{\prime}}^{0 \nu^{\prime}} \\
\vdots \\
g_{N m^{\prime}}^{1 \nu^{\prime}}
\end{array}\right), \mathbf{g}^{1}=
\end{gathered}
$$

From Eqs. (6) and (7), we obtain

$$
\mathcal{G}=(I-M)^{-1} \mathbf{g},
$$

where $I$ is $2(N+1) \times 2(N+1)$ unit matrix and

$$
M=\left[\begin{array}{ll}
M^{11} & M^{12} \\
M^{21} & M^{22}
\end{array}\right]
$$

with $M^{11}, M^{12}, M^{21}$ and $M^{22}$ are $(N+1) \times(N+1)$ matrices whose matrix elements are as follows

$$
\begin{aligned}
M_{m m^{\prime}}^{11}= & \left(a_{m} f_{\mathbf{k}+m \mathbf{Q}}^{*}+b_{m} g_{\mathbf{k}+m \mathbf{Q}}^{*}\right) \delta_{m+1 m^{\prime}} \\
& +\left(a_{m} f_{\mathbf{k}+(m-1) \mathbf{Q}}+b_{m} g_{\mathbf{k}+(m-1) \mathbf{Q}}\right) \delta_{m-1 m^{\prime}} \\
M_{m m^{\prime}}^{12}= & \left(a_{m} g_{\mathbf{k}+m \mathbf{Q}}^{*}-b_{m} f_{\mathbf{k}+m \mathbf{Q}}^{*}\right) \delta_{m+1 m^{\prime}} \\
& +\left(a_{m} g_{\mathbf{k}+(m-1) \mathbf{Q}}-b_{m} f_{\mathbf{k}+(m-1) \mathbf{Q}}\right) \delta_{m-1 m^{\prime}} \\
M_{m m^{\prime}}^{21}= & \left(b_{m} f_{\mathbf{k}+m \mathbf{Q}}^{*}+c_{m} g_{\mathbf{k}+m \mathbf{Q}}\right) \delta_{m+1 m^{\prime}} \\
& +\left(b_{m} f_{\mathbf{k}+(m-1) \mathbf{Q}}+c_{m} g_{\mathbf{k}+(m-1) \mathbf{Q}}\right) \delta_{m-1 m^{\prime}} \\
M_{m m^{\prime}}^{22}= & \left(b_{m} g_{\mathbf{k}+m \mathbf{Q}}^{*}-c_{m} f_{\mathbf{k}+m \mathbf{Q}}^{*}\right) \delta_{m+1 m^{\prime}} \\
& +\left(b_{m} g_{\mathbf{k}+(m-1) \mathbf{Q}}-c_{m} f_{\mathbf{k}+(m-1) \mathbf{Q}}\right) \delta_{m-1 m^{\prime}}
\end{aligned}
$$

We note that the charged modulations observed in STM experiments are weak, i.e. $f_{\mathbf{k}+m \mathbf{Q}}$ and $g_{\mathbf{k}+m \mathbf{Q}}\left(\right.$ or $\left.\lambda^{\prime} s\right)$ are small. Expanding Eq. (10), we have $\mathcal{G}=(I+M+$ $\left.M^{2}+\cdots\right)$ g. From Eq. (7), we finally obtain the green's functions

$$
\begin{aligned}
G_{m m^{\prime}}^{\nu \nu^{\prime}}\left(\mathbf{k}, \mathbf{k}^{\prime} ; i \omega_{n}\right) & \left.=\sum_{\mathcal{G}_{m m^{\prime \prime}}^{\nu^{\prime \prime}}}(-1)^{\left(1-\nu^{\prime \prime}\right) \nu} \xi_{\mathbf{k} m \nu+\nu^{\prime \prime}} ; i \omega_{n}\right) \\
&
\end{aligned}
$$

To compare with the STM experiments, up to the first order in $\lambda^{\prime}$ s, we derive the local density of states

$$
\begin{aligned}
& \rho(\mathbf{r}, \omega)=-\left.\frac{1}{\pi} \operatorname{Im} \sum_{\sigma}\left[-\mathcal{F}<c_{\mathbf{r} \sigma}(\tau) c_{\mathbf{r} \sigma}^{\dagger}(0)>\right]\right|_{i \omega_{n} \rightarrow \omega+i 0^{+}} \\
& \quad=-\frac{2}{\mathcal{N} \pi} \operatorname{Im} \sum_{\mathbf{k} m}\left\{a_{m}\left(\mathbf{k} ; i \omega_{n}\right)+\left(f_{\mathbf{k}+m} \mathbf{Q} e^{i \mathbf{Q} \cdot \mathbf{r}}+c . c\right)\right. \\
& \quad \times\left[a_{m}\left(\mathbf{k} ; i \omega_{n}\right) a_{m+1}\left(\mathbf{k} ; i \omega_{n}\right)-b_{m}\left(\mathbf{k} ; i \omega_{n}\right) b_{m+1}\left(\mathbf{k} ; i \omega_{n}\right)\right] \\
& \quad+\left(g_{\mathbf{k}+m} \mathbf{Q} e^{i \mathbf{Q} \cdot \mathbf{r}}+c . c\right)\left[a_{m}\left(\mathbf{k} ; i \omega_{n}\right) b_{m+1}\left(\mathbf{k} ; i \omega_{n}\right)\right. \\
& \left.\left.\quad+b_{m}\left(\mathbf{k} ; i \omega_{n}\right) a_{m+1}\left(\mathbf{k} ; i \omega_{n}\right)\right]\right\}\left.\right|_{i \omega_{n} \rightarrow \omega+i 0^{+}}
\end{aligned}
$$

where $\mathcal{N}$ is the number of sites in the lattice and $c_{\mathbf{r} \sigma}=$ $\mathcal{N}^{-1 / 2} \sum_{\mathbf{k} m} c_{\mathbf{k}+m \mathbf{Q} \sigma} e^{i(\mathbf{k}+m \mathbf{Q}) \cdot \mathbf{r}}$. Obviously, the first term in Eq. (14) is nothing but LDOS for superconducting state. The other terms are the LDOS modulations with period $2 \pi / \mathrm{Q}$ due to the hopping and pairing modulations, respectively. The Fourier component of LDOS at the ordering wavevector $\mathbf{Q}$ is

$$
\begin{aligned}
\rho_{\mathbf{Q}}(\omega)= & \frac{1}{\mathcal{N}} \sum_{\mathbf{r}} e^{-i \mathbf{Q} \cdot \mathbf{r}} \rho(\mathbf{r}, \omega) \\
= & -\frac{2}{\mathcal{N} \pi} \sum_{\mathbf{k} m}\left\{f _ { \mathbf { k } + m \mathbf { Q } } \operatorname { I m } \left[a_{m}\left(\mathbf{k} ; i \omega_{n}\right) a_{m+1}\left(\mathbf{k} ; i \omega_{n}\right)\right.\right. \\
& \left.-b_{m}\left(\mathbf{k} ; i \omega_{n}\right) b_{m+1}\left(\mathbf{k} ; i \omega_{n}\right)\right] \\
& +g_{\mathbf{k}+m \mathbf{Q}} \operatorname{Im}\left[a_{m}\left(\mathbf{k} ; i \omega_{n}\right) b_{m+1}\left(\mathbf{k} ; i \omega_{n}\right)\right. \\
& \left.\left.+b_{m}\left(\mathbf{k} ; i \omega_{n}\right) a_{m+1}\left(\mathbf{k} ; i \omega_{n}\right)\right]\right\}\left.\right|_{i \omega_{n} \rightarrow \omega+i 0^{+}}
\end{aligned}
$$

It is clear that when several of the charge density wave orders exist simultaneously, the total $\rho_{\mathbf{Q}}(\omega)$ is obtained by a superposition of those of them.

In Fig. 1, we show the real parts of $\rho_{\mathbf{Q}}(\omega)$ for different charge density wave orders in $\mathrm{Bi}_{2} \mathrm{Sr}_{2} \mathrm{CaCu}_{2} \mathrm{O}_{8+\delta}$, whose imaginary parts are zero or are proportional to its real parts. Obviously, the experimental curves (Fig. 3) of Ref. [2] cannot be explained by the site- or bond-centered charge modulation alone (Fig.1a). But our results for the dimerization hopping (Fig. 1b, c) and transverse pairing modulations (Fig. 1e) are consistent with the STM experiments. Because the imaginary part of $\rho_{\mathbf{Q}}(\omega)$ 
observed in the STM experiments is small, we conclude that the charge stripes are mainly formed by the transverse dimerization hopping and transverse pairing modulations. We note that our results are somewhat different from those of Ref. [8], this may be due to the approximations using in Ref. [8]. We also note that the charge modulations discussed in Ref. [9] are two-dimensional but ours are one-dimensional.

Fig. 2a-e show $\operatorname{Re} \rho_{\mathbf{Q}}(\omega)$ for $\mathrm{YBa}_{2} \mathrm{Cu}_{3} \mathrm{O}_{6.35}$ with the same charge density wave orders as in Fig. 1 . The curves in Fig. 1 and Fig. 2 are very different due to the different ordering wavevector and doping in two superconductors. For the dimerization hopping modulations (Fig. 2 b, c), $\operatorname{Re} \rho_{\mathbf{Q}}(\omega)$ has single peak rather than two peaks. For the pairing modulations (Fig. $2 \mathrm{~d}$, e), there exist a strong peak and a weak peak at symmetric positions. These results are expected to be verified by the STM experiments.

Finally, we discuss the ARPES experiments on the charge stripes, which measure the spectral function. From Eqs. (3) and (13), we obtain the spectral function up to the second order correction

$$
\begin{aligned}
& A_{\mathbf{K}}(\omega) \equiv A_{m}(\mathbf{k}, \omega) \\
& =-\left.\frac{1}{\pi} \operatorname{Im}\left[-\mathcal{F}<c_{\mathbf{k}+m \mathbf{Q} \uparrow}(\tau) c_{\mathbf{k}+m \mathbf{Q} \uparrow}^{\dagger}(0)>\right]\right|_{i \omega_{n} \rightarrow \omega+i 0^{+}} \\
& =-\frac{1}{\pi} \operatorname{Im} a_{m}\left(\mathbf{k} ; i \omega_{n}\right)\left\{1+\sum_{\nu}\left[a_{m+\nu-1}\left(\mathbf{k} ; i \omega_{n}\right) f_{\mathbf{k}+(m+\nu-1) \mathbf{Q}}^{*}\right.\right. \\
& \left.+b_{m+\nu-1}\left(\mathbf{k} ; i \omega_{n}\right) g_{\mathbf{k}+(m+\nu-1) \mathbf{Q}}^{*}\right]\left[a_{m+\nu}\left(\mathbf{k} ; i \omega_{n}\right) f_{\mathbf{k}+(m+\nu-1) \mathbf{Q}}\right. \\
& \left.+b_{m+\nu}\left(\mathbf{k} ; i \omega_{n}\right) g_{\mathbf{k}+(m+\nu-1) \mathbf{Q}}\right]+\left[b_{m-1}\left(\mathbf{k} ; i \omega_{n}\right) f_{\mathbf{k}+(m-1) \mathbf{Q}}^{*}\right. \\
& \left.+c_{m-1}\left(\mathbf{k} ; i \omega_{n}\right) g_{\mathbf{k}+(m-1) \mathbf{Q}}^{*}\right]\left[a_{m}\left(\mathbf{k} ; i \omega_{n}\right) g_{\mathbf{k}+(m-1) \mathbf{Q}}\right. \\
& \left.-b_{m}\left(\mathbf{k} ; i \omega_{n}\right) f_{\mathbf{k}+(m-1) \mathbf{Q}}\right]+\left[b_{m+1}\left(\mathbf{k} ; i \omega_{n}\right) f_{\mathbf{k}+m \mathbf{Q}}\right. \\
& \left.+c_{m+1}\left(\mathbf{k} ; i \omega_{n}\right) g_{\mathbf{k}+m \mathbf{Q}}\right]\left[a_{m}\left(\mathbf{k} ; i \omega_{n}\right) g_{\mathbf{k}+m \mathbf{Q}}^{*}\right. \\
& \left.\left.-b_{m}\left(\mathbf{k} ; i \omega_{n}\right) f_{\mathbf{k}+m \mathbf{Q}}^{*}\right]\right\}\left.\right|_{i \omega_{n} \rightarrow \omega+i 0^{+}} \\
& \equiv A_{0 \mathbf{K}}(\omega)+\delta A_{\mathbf{K}}(\omega)
\end{aligned}
$$

The first term in the above equation is the spectral function of the superconducting state. The others are those due to the weak charge modulations, which are the second order small quantities. Obviously, the spectral functions $A_{\mathbf{K}}(\omega)$ for different hopping (pairing) modulations have similar energy dependence at the same momentum $\mathbf{K}$. So it is difficult to clarify which kinds of hopping (pairing) modulations the charged stripes belong by the ARPES experiments. However, the hopping and pairing modulations have unique energy dependence for $A_{\mathbf{K}}(\omega)$ which could be distinguished experimentally. Fig. 3 and Fig. 4 show the spectral functions $A_{\mathbf{K}}(\omega)$ at various momenta $\mathbf{K}$ for $\mathrm{Bi}_{2} \mathrm{Sr}_{2} \mathrm{CaCu}_{2} \mathrm{O}_{8+\delta}$ and $\mathrm{YBa}_{2} \mathrm{Cu}_{3} \mathrm{O}_{6.35}$ with hopping and pairing modulations, respectively.

In summary, we study the effects of various charge density wave orders on the d-wave superconductor. We conclude that the dimerization hopping and transverse pairing modulations and experimental results are in excellent agreement. The origin of such charge modulations is due to the hopping and gap disorders in the superconductor, which will be investigated in detail in another paper [12].
We also discuss the ARPES experiments on the charged stripes, which can distinguish the hopping and pairing modulations.

The author wish to thank Prof. C. S. Ting for useful discussions. This work has been supported by the Texas Center for Superconductivity at the University of Houston and by the Robert A. Welch Foundation.

[1] J. E. Hoffman, E. W. Hudson, K. M. Lang, V. Madhavan, H. Eisaki, S. Uchida, J. C. Davis, Science 295, 466 (2002).

[2] C. Howald, H. Eisaki, N. Kaneko, A. Kapitulnik, condmat/0201546.

[3] H. A. Mook, P. Dai, and F. Dogan, Phys. Rev. Lett. 88, 97004 (2002).

[4] J-X. Zhu, I. Martin, and A. R. Bishop, condmat/0201519.

[5] A. Polkovnikov, M. Vojta, and S. Sachdev, condmat/0203176.

[6] Y. Chen, H. Y. Chen, and C. S. Ting, cond-mat/0203283.

[7] H. D. Chen, J. P. Hu, S. Capponi, E. Arrigoni, S. C. Zhang, cond-mat/0203332.

[8] D. Podolsky, E. Demler, K. Damle, and B. I. Halperin, cond-mat/0204011.

[9] M. Vojta, cond-mat/0204284.

[10] M. Franz, D. E. Sheehy, and Z. Tesanovic, Phys. Rev. Lett. 88, 257005 (2002).

[11] M. R. Norman, M. Randeria, H. Ding, and J. C. Campuzano, Phys. Rev. B 52, 615 (1994).

[12] Degang Zhang and C. S. Ting, cond-mat/0209318.

FIG. 1. Energy dependence $\operatorname{Re} \rho_{\mathbf{Q}}(\omega)$ of the Fourier component of the LDOS at $\mathbf{Q}=(\pi / 2,0)$ for $\mathrm{Bi}_{2} \mathrm{Sr}_{2} \mathrm{CaCu}_{2} \mathrm{O}_{8+\delta}$ with different charge density wave orders. (a) A site- or bond-centered charge density wave. (b) A longitudinal dimerization charge density wave. (c) A transverse dimerization charge density wave. (d) A longitudinal pairing modulation. (e) A transverse pairing modulation. In both (b) and (d), $\operatorname{Re} \rho_{\mathbf{Q}}(\omega)$ was multiplied by -1 to compare conveniently with the experiments.

FIG. 2. $\operatorname{Re} \rho_{\mathbf{Q}}(\omega)$ at $\mathbf{Q}=(\pi / 4,0)$ for $\mathrm{YBa}_{2} \mathrm{Cu}_{3} \mathrm{O}_{6.35}$ with the same charge density wave orders as in Fig. 1.

FIG. 3. Spectral function $A_{\mathbf{K}}(\omega)$ vs $\omega$ at various $\mathbf{K}$ for $\mathrm{Bi}_{2} \mathrm{Sr}_{2} \mathrm{CaCu}_{2} \mathrm{O}_{8+\delta}$ with hopping and pairing modulations.

FIG. 4. Spectral function $A_{\mathbf{K}}(\omega)$ vs $\omega$ at various $\mathbf{K}$ for $\mathrm{YBa}_{2} \mathrm{Cu}_{3} \mathrm{O}_{6.35}$ with hopping and pairing modulations. 

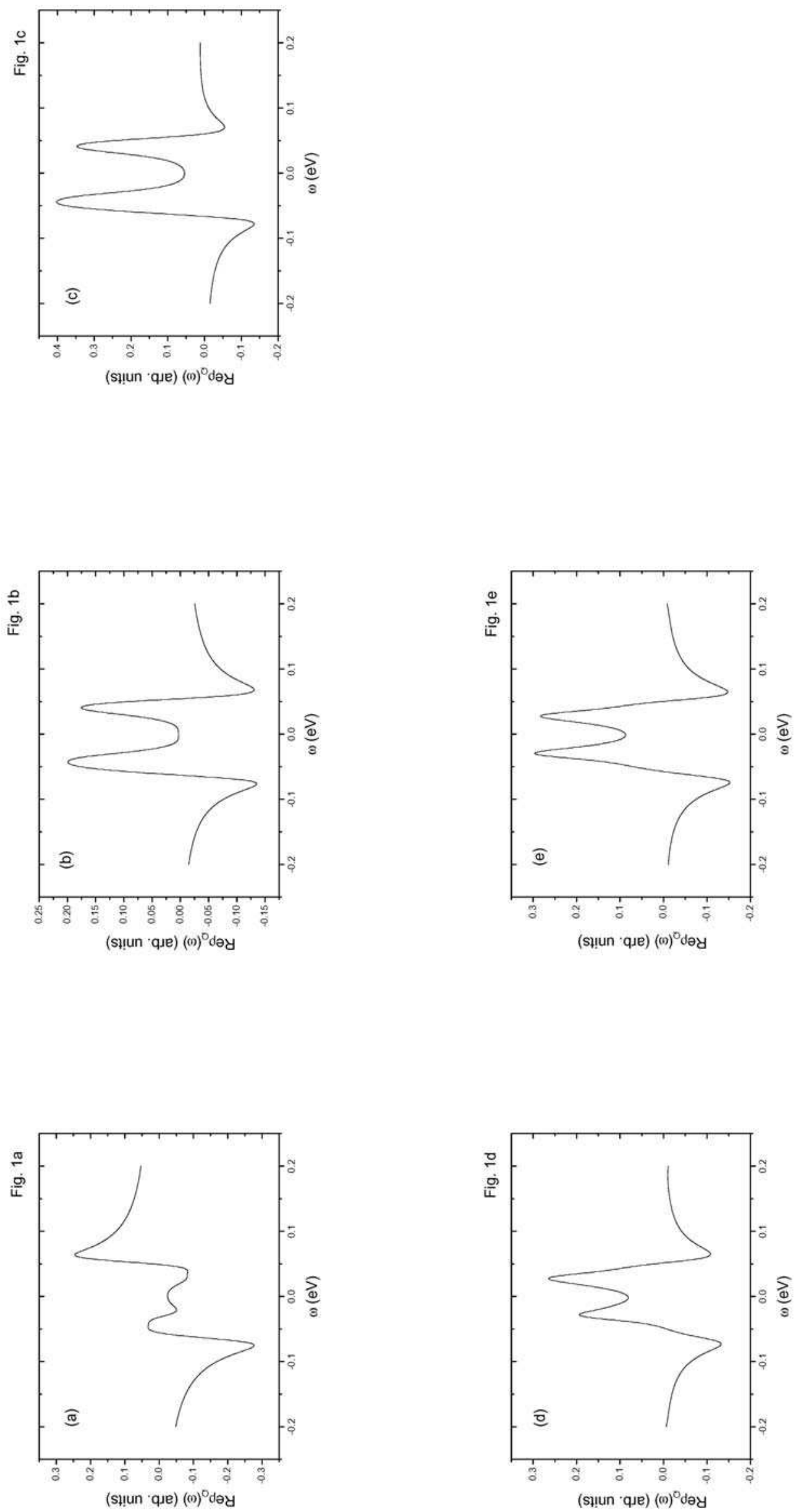

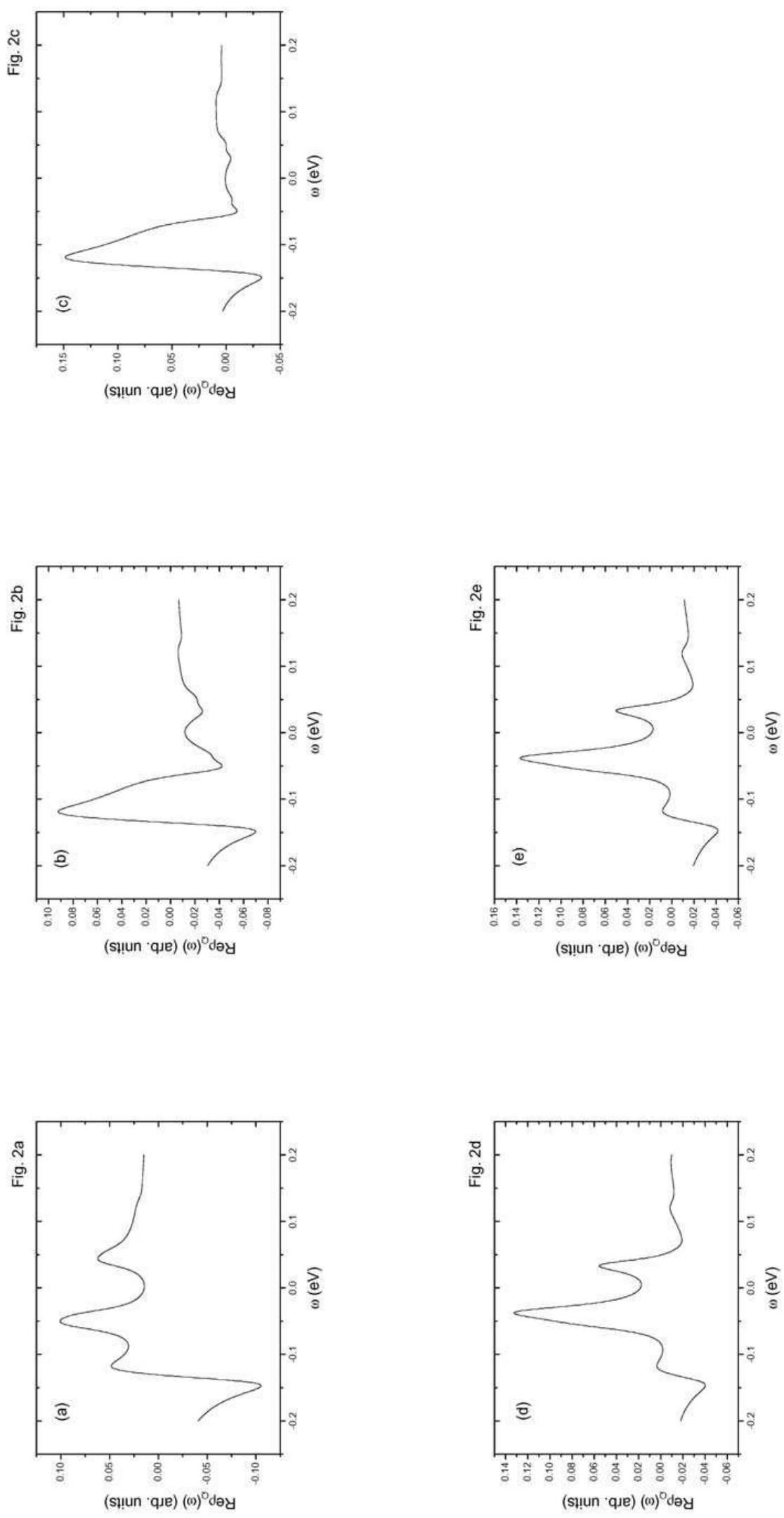

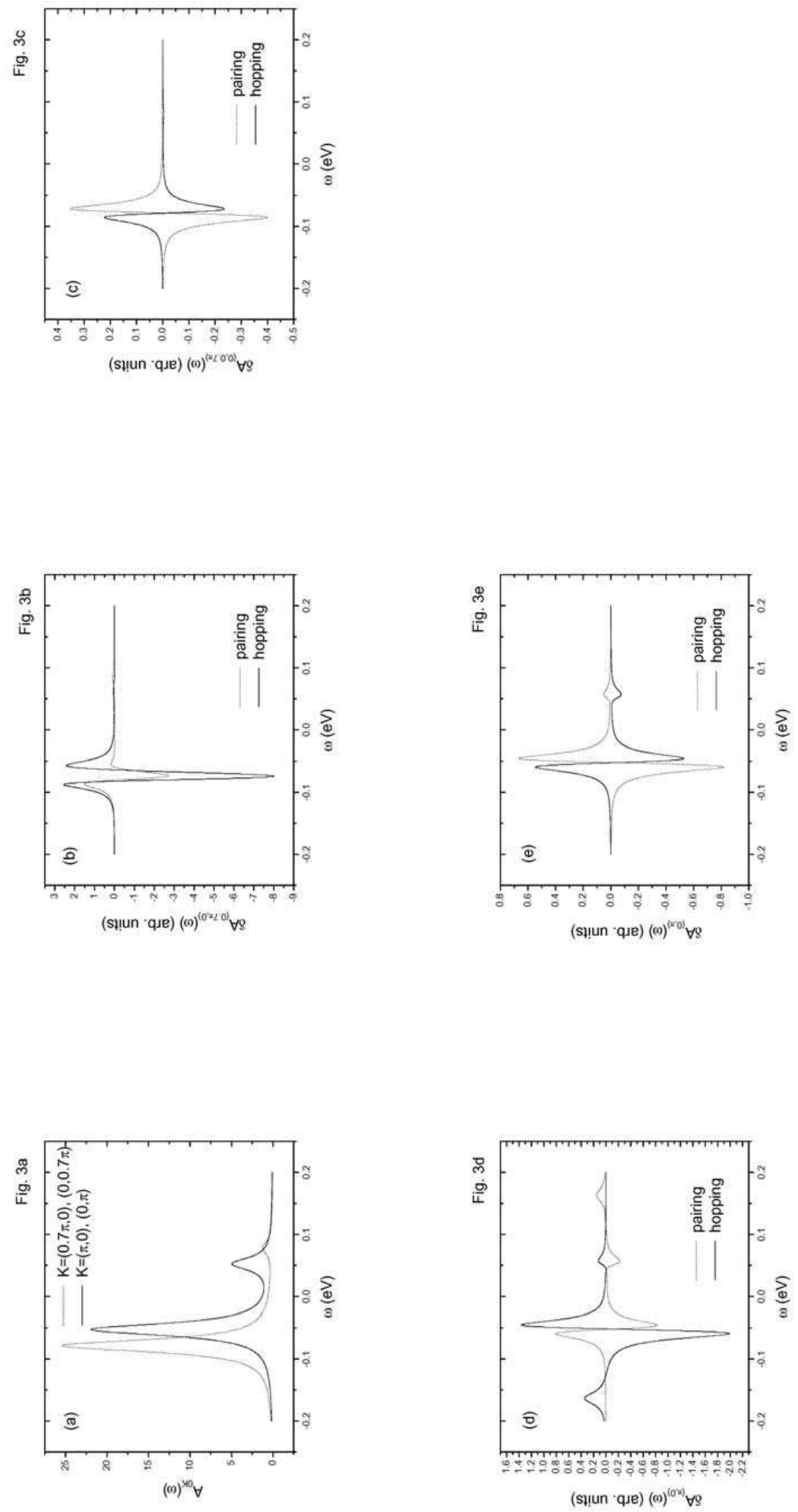

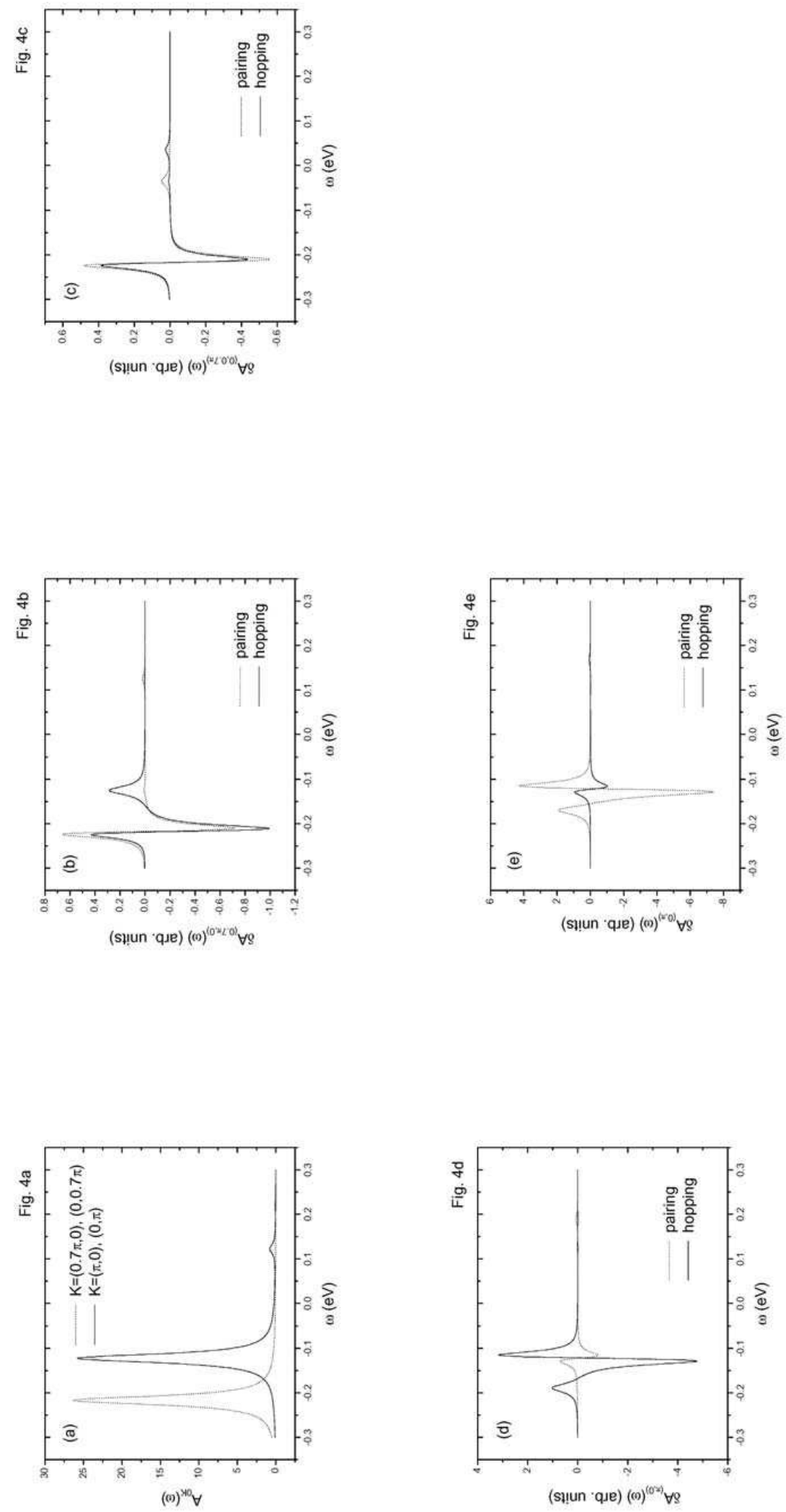